\title{
Balance y recomendaciones sobre salud mental de la población y del personal de salud durante la pandemia de COVID-19 en el Perú
}

\section{Balance and recommendations on the mental health of the population and health personnel during the COVID-19 pandemic in Peru}

\author{
Javier E. Saavedra'
}

Saavedra, J. Balance y recomendaciones sobre salud mental de la población y del personal de salud durante la pandemia de COVID- 19 en el Perú . Rev Soc Peru Med Interna. 2020;33(4): I 35-I 37.

https://doi.org/10.36393/spmi.v33i4.559

empeore durante y posterior a la pandemia, y siguiendo experiencias internacionales se espera que estos problemas se incrementen en 2 a 4 veces en comparación de periodos sin pandemia. ${ }^{6}$ En el momento se encuentran en marcha distintas investigaciones sobre prevalencia de problemas de salud mental en el país, tanto a nivel poblacional como de trabajadores de la salud, aunque al momento de la redacción de este documento no se cuenta todavía con resultados publicados. No obstante, la Dirección de Salud Mental del MINSA (DSM-MINSA) ha adelantado hallazgos preliminares de un estudio poblacional, donde participaron 58349 personas, y dio cuenta que el 28,5\% de los encuestados reunía criterios mínimos para episodio depresivo y de este grupo $41 \%$ era de una severidad de moderada a severa; además, $12,8 \%$ refirió ideación suicida. ${ }^{7}$ Asimismo, se adelantó que $68 \%$ estaría sufriendo algún tipo de problema o sintomatología relacionada con la salud mental, entre los cuales resaltaron $55,7 \%$ de problemas para dormir, $42,8 \%$ problemas de apetito, $44 \%$ cansancio o falta de energía, y $35,5 \%$ falta de concentración. Un estudio preliminar reciente en 400 personas, a través de cuestionarios en línea, reportó que $35,5 \%$ de los adultos de Lima Metropolitana presentaba al menos un nivel de estrés leve, y 9,3\% presentó estrés severo. ${ }^{8}$ Están en marcha otras investigaciones del MINSA para determinar el nivel de cobertura de las necesidades de atención en salud mental por el Instituto Nacional de Salud Mental.

Mención aparte merecen los problemas de violencia, en general, y violencia intrafamiliar, en particular, lo cual la ONU ha instado a que los gobiernos se ocupen de este 
problema en sus planteamientos de respuestas contra el COVID-19. ${ }^{9}$ En este sentido, el Ministerio de la Mujer ha reportado un incremento de los casos de violencia contra la mujer según los reportes de los Centros de Emergencia Mujer y las líneas de ayuda con respecto a periodos similares en el año 2019. ${ }^{10}$

Por otro lado, desde la oferta, esta pandemia ha desnudado las serias deficiencias y descuidos del sector salud que arrastra desde hace décadas, con presupuestos asignados a la salud mental por debajo del promedio latinoamericano. Durante la pandemia, la atención psiquiátrica se ha visto seriamente restringida. Sin embargo, el MINSA ha promovido la continuidad de atención para emergencias, la implementación de equipos de atención para casos moderados a severos a través de seguimientos virtuales, vistas domiciliarias, intervención de poblaciones en riesgo y acompañamiento psicosocial a trabajadores en la primera línea de enfrentamiento de la pandemia (personal de salud, personas de la fuerzas armadas y policiales). ${ }^{11}$

El Ministerio de Salud ha venido implementando en los últimos años la Reforma de la Salud Mental con enfoque comunitario, habiéndose organizado más de 150 Centros de Salud Mental Comunitarios de tipo multidisciplinario (CSMC) en todo el país, con el objetivo de lograr 281 para el próximo año, en el intento de lograr un centro de salud mental comunitario para cada 100000 habitantes. ${ }^{12} \mathrm{La}$ presencia de estos CSMC ha servido impensadamente para enfrentar de mejor manera los efectos en la salud mental de esta pandemia. Informes oficiales del MINSA han dado cuenta en el que en los cuatro primeros meses de pandemia se han atendido 44129 casos en estos CSMC, de los cuales $36,1 \%$ fue por ansiedad, 16,0\% fue depresión y $6,7 \%$ fue por problemas de adicciones, entre otras.

Otra de las herramientas que se viene implementado es la atención virtual a través de telesalud, de la cual existen evidencias internacionales que sería tan efectivas como las atenciones directas. ${ }^{13}$ En este sentido, el MINSA informó que, entre mayo y agosto del presente año, estos mismos centros han podido atender 22204 pacientes a través de la telemedicina en todo el país. ${ }^{14}$ Los problemas por considerar en este sentido son las limitaciones que podría tener algunos grupos vulnerables para acceder a esta tecnología como los adultos mayores o las personas con limitaciones económicas o en situación de pobreza. ${ }^{15}$. Además, desde el inicio de la pandemia se implementó una línea de ayuda 24 x 7 para cualquier consulta relacionada a la salud mental o información sobre el CSMC más cercano al hogar de la persona, y se han atendido durante la pandemia hasta setiembre 2020 más de 73000 orientaciones, el 71,4\% relacionadas a estrés, el $17,9 \%$ por síntomas ansiosos o depresivos, 3,6\% por síntomas o diagnósticos psiquiátrico, el $2,4 \%$ por conflictos personales y familiares y $1,2 \%$ por problemas sentimentales. ${ }^{16}$

El MINSA ha emitido, además, tres documentos técnicos para reducir el impacto de la pandemia en la salud mental. Uno dirigido a la población general estableciendo que todas las instituciones prestadoras públicas o privadas deben incorporar el cuidado de la salud mental en su planes y estrategias de intervención frente la pandemia a través de las redes comunitarias, precisándose los procedimientos para fomentar el autocuidado de la población identificando oportunamente y diagnosticando a las personas con problemas de salud mental, en especial ansiedad depresión y problemas de alcoholismo. ${ }^{17}$ En este sentido, las instituciones psiquiátricas han implementado sistemas de atención virtual tanto para sus pacientes continuadores como para pacientes nuevos. El segundo documento está dirigido al personal de salud reconociendo el importante riesgo de presentar problemas de salud mental, describiendo los lineamientos de cuidado y autocuidado, estrategias de soporte psicosocial disponibles en la institución de salud y las estrategias de identificación e intervención; las disposiciones incluyen la asignación de responsabilidades a nivel central, regional y local, y la designación de un Equipo de Acompañamiento Psicosocial para el Personal de Salud en cada establecimiento, precedido de un diagnostico situacional. ${ }^{18}$ Finalmente, en junio 2020 se emitió el Plan de Salud Mental en el Contexto de COVID-19, con el objetivo de "reducir el impacto de la pandemia del COVID-19 en la salud mental de la población, fortaleciendo sus recursos individuales y comunitarios de afrontamiento y atendiendo a las personas con problemas mentales en el contexto de la crisis económica y social derivada de ella", el cual integra y organiza las estrategias arriba mencionadas en distinto objetivos específicos.

\section{Recomendaciones}

Todo los Estados, en especial los latinoamericanos, deben comprometerse en alcanzar un presupuesto mínimo asignado a salud y a la salud mental para reducir riesgos de futuras pandemia o situaciones de emergencia sanitaria. Debemos prepararnos para otras pandemias e instar a los gobiernos a proseguir con la Reforma de la Salud Mental con enfoque comunitario donde existiere o implementarlas donde no.

Brindar atención y soporte a los trabajadores de la salud, pero tomando en consideración los puntos de vista de los mismo y no desde estrategias impuestas desde afuera.

Establecer y afianzar medidas generales para reducción de estrés en la comunidad latinoamericana como: asegura provisiones de necesidades básicas para las personas de menores recursos, incrementar y fomentar la comunicación a través de las redes sociales.

Reducir el impacto de la pandemia en las personas contagiadas mejorando u optimizando la comunicación entre personas aisladas por el internamiento y sus familiares Optimizar los recursos informáticos de telesalud mental tomado en consideración necesitad de ampliar la alfabetización informática a sectores vulnerables de la población.

Promover más intensamente el autocuidado a través de las redes y los medios de comunicación masiva a través del gobierno central y gobiernos locales

Revalorar y resaltar en la comunidad los valores culturales 
latinoamericanos alrededor de la familia, la solidaridad y el optimismo en un futuro mejor.

Revalorar conceptos de la medicina centrada en las personas en contexto de la persona que es afectada por el virus, su familia y el personal de salud.

Apoyar la investigación en salud mental para que las intervenciones tengan sustento en la evidencia.

\section{REFERENCIAS BIBLIOGRÁFICAS}

I. Decreto Supremo N064-2020-PCM que prorroga el Estado de Emergencia Nacional por las graves circunstancias que afectan la vida de la nación a consecuencia del COVID-19 y dicta otras medidas.

2. Ping W, Zheng J, Niu X, Guo C, Zhang J, Yang H, Shi Y. Evaluation of health-related quality of life using EQ-5D in China during the COVID-19 pandemic. PLoS ONE. 2020; I5(6): e0234850. https://doi. org/I0.137//journal.pone.0234850

3. Rothstein MA, Talbott MK. Encouraging compliance with quarantine: a proposal to provide job security and income replacement. Am J Public Health. 2007;97 Suppl I(Suppl I):S49-S56. doi: I0.2105/ AJPH.2006.097303

4. Blake KD, Blendon RJ, Viswanath K. Employment and compliance with pandemic influenza mitigation recommendations. Emerg Infect Dis. 2010 Feb; |6(2):2 I2-8. doi: I0.320I/eid |602.090638

5. Suarez Ognio L, Miranda Monzón J, Valdez Huarcaya W, Driver C. Carga de Enfermedad en el Perú. Estimación de los años de vida saludables perdidos 2016. Lima:Dirección General de Epidemiología, MINSA; diciembre 2018.

6. Czeisler MÉ, Lane RI, Petrosky E, et al. Mental health, substance use, and suicidal ideation during the COVID-1 9 pandemic - United States, June 24-30, 2020. MMWR. 2020;69:1049-57. http://dx.doi. org/ $/ 0.15585 / \mathrm{mmwr} . \mathrm{mm} 6932 \mathrm{a}$ I

7. MINSA. Plan de Salud Mental Perú, 2020-202I (En el contexto COVID-19). Lima: MINSA; Julio 2020. URL disponible en: https://cdn www.gob.pe/uploads/document/file/8 I I |38/Plan_de_Salud_ mental .PDF

8. Krüger-Malpartida H, Pedraz-Petrozzi B, Arevalo-Flores M, Salmavides-Cuba F, Anculle-Arauco V, Dancuart-Mendoza M Effects on the mental health after COVID-19 lockdown period: results from a population survey study in Lima, Peru. 22 September 2020, Preprint (Version I). Research Square. URL disponible en: https://www.researchsquare.com/article/rs-8||| $7 / \mathrm{vl}$

9. The Department of Global Communication - United Nations. Víctimas de la violencia doméstica atrapadas durante la pandemia. Publicación Internet. URL disponible en: https://www.un.org/es/ coronavirus/articles/un-supporting-trapped-domestic-violencevictims-during-covid-19-pandemic

10. Ministerio de la Mujer y Poblaciones Vulnerables (MIMP) Estadísticas del Programa Nacional para la Prevención y Erradicación de la Violencia contra las Mujeres e Integrantes del Grupo Familiar -AURORA- Perú, 2020. URL disponible en: https://www.mimp.gob. pe/contigo/contenidos/pncontigo-articulos.php?codigo $=31$

II. Cutipé Y. Documento Técnico: Plan de Salud Mental en el Contexto del COVID-19. Youtube, subido por la Dirección General de Telesalud Transferencias y Urgencias. Lima: Dirección de Salud Mental de la Dirección General de Intervenciones Estratégicas en Salud Publica, MINSA; 19 junio 2020. URL disponible en: https:// www.youtube.com/watch?v=AEcW puGBUoc.

12. Castillo-Martell H, Cutipé-Cárdenas Y. Implementación, resultados iniciales y sostenibilidad de la reforma de servicios de salud mental en el Perú, 2013-2018. Rev Peru Med Exp Salud Publica. 2019;36(2):326-33. URL disponible en: http://dx.doi.org//0.17843/ rpmesp.2019.362.4624

13. Hubley S, Lynch SB, Schneck C, Thomas M, Shore J. Review of key telepsychiatry outcomes. World J Psychiatry. 2016;6(2):269-282. Published 2016 Jun 22. doi:I0.5498/wjp.v6.i2.269

14. MINSA. Centros de Salud Mental Comunitarios han brindado 431 795 atenciones a través del servicio de telemedicina. Nota de Prensa. URL disponible en: https://www.gob.pe/institucion/minsa/ noticias/30255 I-centros-de-salud-mental-comunitarios-hanbrindado-43I-795-atenciones-a-traves-del-servicio-de-telemedicina

15. O'Brien M, McNicholas F. The use of telepsychiatry during COVID-19 and beyond. Ir J Psychol Med. 2020 May 21:I-6. doi: 10.1017/ipm.2020.54. Epub ahead of print.

16. MINSA. Más de 84 mil orientaciones en salud mental atendió el Minsa a través de la Línea I I 3 Salud en 2020. Nota de Prensa. URL disponible en: https://www.gob.pe/institucion/minsa/ noticias/30053 I-mas-de-84-mil-orientaciones-en-salud-mentalatendio-el-minsa-a-traves-de-la-linea- I | 3-salud-en-2020

17. MINSA. Guía técnica. Cuidado de la salud mental de la población afectada, familias y comunidad, en el contexto del COVID-19. Resolución Ministerial $\mathrm{N}^{\circ}$ 186-2020-MINSA. URL disponible en: http://bvs.minsa.gob.pe/local/MINSA/500I.pdf

18. MINSA. Guía técnica. Cuidado de la salud mental del personal de salud, en el contexto del COVID-19. Resolución Ministerial $N^{\circ}$ 180-2020-MINSA. URL disponible en: http://bvs.minsa.gob.pe/ local/MINSA/5000.pdf

Lima, 27 de noviembre del 2020.

CORRESPONDENCIA:

Javier E. Saavedra, jesaavedra@outlook.es 